\title{
eReefs - a new perspective on the Great Barrier Reef
}

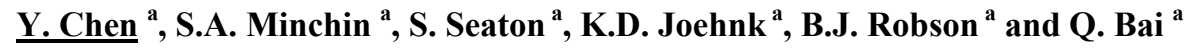 \\ ${ }^{a}$ CSIRO Land and Water, Canberra ACT 2601, Australia \\ Email:yun.chen@,csiro.au
}

\begin{abstract}
The Great Barrier Reef (GBR) in Queensland of Australia is recognised globally for its beauty and biodiversity. Managing the GBR is a complex challenge. Its managers grapple with multiple threats to its future across spatial and temporal scales. In order to provide the step change that is needed to support regulatory, planning and management decisions, a project called eReefs has been proposed. eReefs is an integrated operational system of data sources, forecasting and hindcasting models and visualisation and reporting services, and will deliver a comprehensive suite of management tools using real-time or near-realtime information across the paddock-to-ocean scale for the GBR. eReefs will transform science and management of the GBR by dramatically improving the transparency, repeatability and application of information, and enable dynamic and auditable knowledge to be utilised by users and managers of the GBR. It will support managers, industries, farm businesses, and the community in monitoring the current status of the GBR environment at a whole of system scale.
\end{abstract}

A small project, the eReefs Pilot, was undertaken to test the eReefs concept and to show the potential of eReefs to deliver a comprehensive suite of management tools for the GBR. The study aimed to demonstrate the potential of linking catchment, lagoon and oceanic models across the GBR environment. It made use of existing data from the Fitzroy WaterCAST catchment model and 3D (time based) polygonal contours from the Fitzroy estuary and whole of GBR (SHOC) hydrodynamic models for running, orchestrating and visualising various scenarios which reanalyzed historical data to explore what actually happened. This paper focuses on an initial step towards realising the full eReefs vision through the description of the following key deliverables of the project:

(1) Development of the eReefs Google Earth Water Quality Visualisation Platform;

(2) Orchestration of the Fitzroy Catchment, Estuary and the whole of GBR Lagoon models in a workflow environment;

(3) Examination and visualisation of a number of catchment, estuary and lagoon scenarios; and

(4) Reconstruction of two historical flood plume events in the Burdekin River and nearby coastal waters.

The eReefs Pilot project has demonstrated the type of functionality that would be possible when the fully operational eReefs is built. It has become a valuable demonstration tool for engaging investors and stakeholders in the aims of eReefs in the longer term. It has also provided an early visualisation platform for immediate rollout where the data and models exist to support the development of the full eReefs vision. The study has highlighted an opportunity to transform our understanding, knowledge and prediction of the GBR environment and ecosystem through a comprehensive and coordinated investment in an operational information platform across spatial and temporal scales for the GBR. It has paved the way for a more robust and detailed development and realisation of the fully operational eReefs in the future.

Keywords: water quality visualisation platform, WaterCAST, SHOC, orchestration, workflow, reconstruction 


\section{INTRODUCTION}

\section{1. eReefs Vision}

The Great Barrier Reef (GBR) is internationally well-known for its beauty and biodiversity (Figure 1). For Australians it is also a source of economic, social and cultural values. The GBR environment is connected to the farms and industries in adjoining river catchments, the towns, communities and industries and ecosystems on the coast which borders it, the Pacific Ocean that surrounds it, and the rapidly changing atmosphere above. Managing the GBR is a complex challenge with multiple threats across spatial and temporal scales,

The 2009 Great Barrier Reef Outlook Report (GBR Marine Park, 2009) identified that the GBR is subject to multiple pressures, including: nutrient, sediment and pesticide loads from catchment degradation, threats associated with anthropogenic climate change, including warming and coral bleaching, changes in ocean circulation and productivity, and ocean acidification, and marine uses such as fishing and tourism. The complexity of issues challenging the GBR have prevented researchers, managers, industry and the community from addressing these pressures and their interactions and consequences for ecosystem health and services, at appropriate scales. Research has tended to address particular pressures and processes in isolation, often at local scales, ignoring interactions among pressures at longer time and larger spatial scales. Recent reviews have highlighted the lack of quantitative understanding of the interactions between catchment processes and GBR health at whole-of-reef scales. An opportunity exists to transform our understanding, knowledge and prediction of the GBR environment and ecosystem through a comprehensive and coordinated investment in an operational information platform for the GBR.

The Great Barrier Reef Foundation, in collaboration with the Australian Institute of Marine Science (AIMS), CSIRO, the Australian Bureau of Meteorology, the Great Barrier Reef Marine Park Authority, the Australian and Queensland Governments, and Telstra, propose to develop eReefs - a transformational capability that seeks to deliver a revolution in the science and management of an ecosystem that can be legitimately characterised as an asset of global significance. eReefs is an integrated operational system of data sources, forecasting and hindcasting models and visualisation and reporting tools, which spans the paddock-to-ocean scale. eReefs will draw on real-time or near-real-time information to report on, account for and predict GBR health. It will support managers, industries, farm businesses, and the community in monitoring the current status of the GBR environment at a whole of system scale.

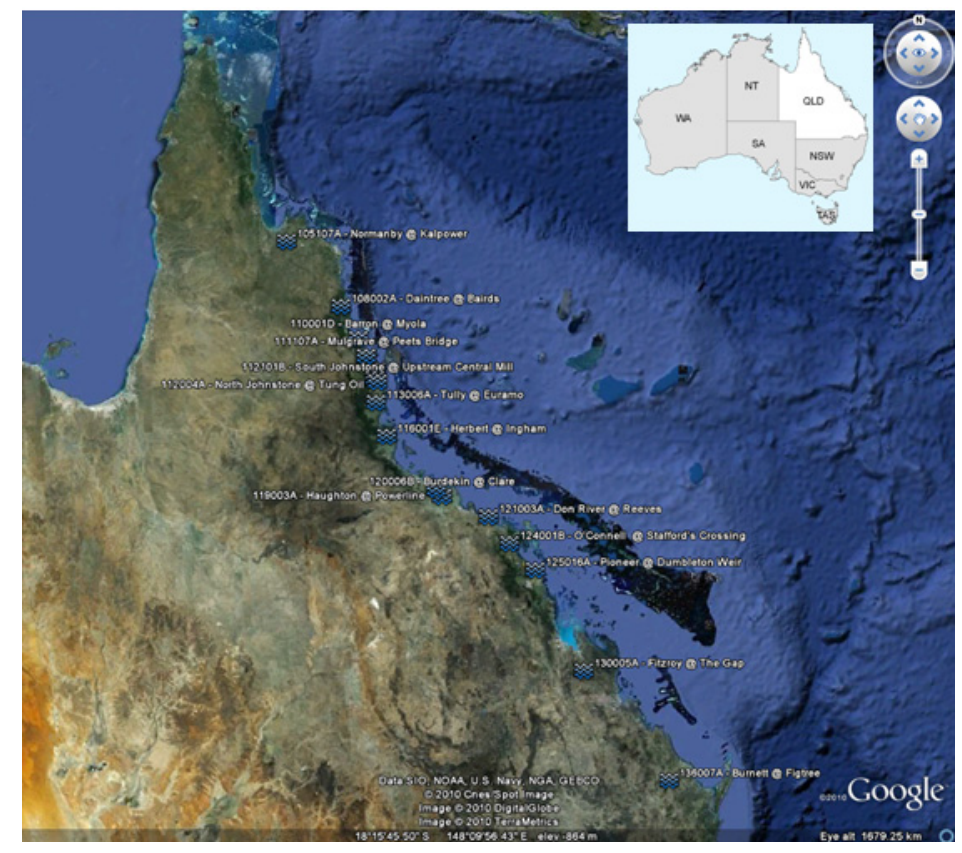

Figure 1. Location of the GBR and its catchments.

\section{2. $\quad$ eReefs Pilot}

In order to test the broader eReefs concept, a small pilot project was undertaken between May and October 2010 to show the potential of eReefs to deliver a comprehensive suite of management tools for the GBR. The Pilot aimed to demonstrate the potential of linking catchment, lagoon and oceanic models across the GBR 
environment. It included Google Earth visualisation of sediment plumes entering the GBR lagoon, coupled with catchment and receiving water modelling of the flood event in key river basins. Driven by real historical data and modelled concentrations for previously recorded flood events and several defined scenarios, and incorporating simplified plume modelling, it was intended to show the potential to visualise the ultimate fate of the sediment released in these events. This paper focuses on an initial step towards realising the full eReefs vision through the description of the following key deliverables of the project:

(1) Development of the eReefs Google Earth Water Quality Visualisation Platform;

(2) Orchestration of the Catchment, Estuary and GBR Lagoon models in a workflow environment;

(3) Examination and visualisation of a number of catchment, estuary and lagoon scenarios; and

(4) Reconstruction of two historical flood plume events in the Burdekin River and nearby coastal waters.

\section{INPUT MODELS}

WaterCAST (Water and Contaminant Analysis and Simulation Tool) and SHOC (Sparse Hydrodynamic Ocean Code) models were used in this study. Examination of the models already developed and calibrated for the GBR catchments, estuaries and lagoon highlighted that the most data and model rich catchment was the Fitzroy River which had a large WaterCAST model developed and calibrated by QLD Department of Environment and Resource Management (DERM). Likewise the Fitzroy estuary had been the focus of a detailed, fine-scale estuary based application of the SHOC model, the same core model that had been used for the development of a $4 \mathrm{~km}$ grid cell model of the entire GBR lagoon developed by CSIRO and AIMS. The Fitzroy was therefore chosen as the focus for demonstration of model orchestration and visualisation tool in the eReefs Pilot.

\subsection{WaterCAST}

WaterCAST is a software product for whole-of catchment modelling. It consists of a node link network for transport of water and constituents within the major channels in a catchment (Cook et al., 2009; eWater CRC, 2010). It is designed to allow modellers and researchers to construct models by selecting and linking component models from a range of available choices. The version of the software used in the eReefs Pilot was a customised version known as the "Dynamic SedNet" version. This software was obtained from the DERM along with a corresponding WaterCAST model representing the most current attempt at modelling the Fitzroy catchment.

The catchment was defined through the use of a digital elevation map, and the models configured largely from SILO rainfall (Jeffrey et al., 2001), and KLSC maps which are key components of Universal Soil Loss Eqution (Searle and Ellis, 2009). For the purpose of the Pilot, the complete Fitzroy model was run at daily time steps from $1 / 1 / 1986$ to $31 / 12 / 2009$. Through the use of the extraction plug-in, the sub period of $10 / 11 / 2008$ to $1 / 4 / 2009$ was then exported for total suspended solids (TSS) and flow at both catchment and node outlets and utilised in both the visualisation tool, and the SHOC modelling.

\section{2. $\mathrm{SHOC}$}

The three-dimensional hydrodynamic model, SHOC (v. 1632), was applied for both the lagoon-scale (4km resolution) model and the estuary-scale receiving water model (the Fitzray Estuary model). SHOC is a finitedifference, baroclinic model based on Blumberg and Herring (1987) and described in detail by Herzfeld et al. (2005). It uses a curvilinear grid, which allows increased grid resolution in areas of more complex morphology, such as along the coast-line. Input data required by SHOC include fresh-water flows from rivers, meteorological conditions across the surface, and surface elevation, temperature and salinity at the seaward boundaries. Outputs from SHOC include three-dimensional fields of velocity, salinity, temperature and water density as well as surface elevation. SHOC outputs are provided as two-dimensional and threedimensional data-blocks in NetCDF format (Rew et al., 1997) at specified time intervals or as time-series in plain-text format at specified points within the model domain.

To allow greater resolution within an area of interest, a higher resolution model can be nested within a regional-scale model. For the Pilot project, we chose to demonstrate this capacity by nesting an extended version of the Fitzroy Estuary model within the lagoon-scale model. The estuary-scale grid has a horizontal resolution ranging from $300 \mathrm{~m}$ in the estuary channel near Rockhampton to $2 \mathrm{~km}$ at its outer boundary. Over most of the domain of the estuary model, the horizontal resolution is less than $1 \mathrm{~km}$ by $1 \mathrm{~km}$. Fresh-water inflow to the Fitzroy Estuary model is taken from output from the Fitzroy Basin WaterCAST model. Outer boundary conditions (salinity, temperature and water velocity) for the estuary-scale model were taken from the output of the lagoon-scale model. 
Both the lagoon-scale model and the Fitzroy Estuary model have been validated in some detail (Herzfeld, 2006; Brando et al., 2007; Robson et al., 2008; Robson and Brando, 2008).

\section{KEY DEVELOPMENT}

\subsection{Water Quality Visualisation Platform}

A key outcome of this project was the delivery of the eReefs Google Earth Water Quality Visualisation Platform (WQVP). This tool was intended to take outputs from the WaterCAST and SHOC models and turn them into Google Earth ready visualisations using Google's Keyhole Markup Language (KML). The visualisations show time-based 3D network data from WaterCAST and time-based 3D polygonal contours from SHOC. Development of this tool was split into two distinct components (Chen et al., 2011). The first component is the eReefGoogleEarthLib that comprises of the routines and classes used to provide a reusable library of classes for generating eReefs related Google Earth KML. The second component is the eReef KML Tool interface project that contains the entire code specific to this incarnation of the user interface (eReefGoogleEarthGenerator). The source code was built using Visual Studio 2008 on a Window XP machine. The interface was enhanced through the use of Microsoft Expression Blend 3.

It should also be noted that while the core requirement to display WaterCAST and SHOC outputs was met, the eReefs KML tool was also designed to accept data from any source that can be manipulated into a simple input format, meaning it may be re-used in many other areas than the GBR.

The visualisation platform developed in this study can display modelled sediment mobilisation events in rivers running into the GBR lagoon and to simulate the spread of sediment plumes in the lagoon (Figure 2).

\subsection{Orchestration Tool}

A significant deliverable of the eReef Pilot was to demonstrate our ability to connect together the WaterCAST and SHOC models, contour generation software, and KML tool into a single executable workflow. This was achieved through the use of a new Microsoft research tool called Trident (Barga et al., 2008). Trident is a Scientific Workflow Workbench which is a set of tools based on the Windows Workflow Foundation that addresses scientists' need for a flexible, powerful way to analyse large, diverse datasets. It includes graphical tools for creating, running, managing, and sharing workflows. Conceptually the workflow from catchment to receiving water was quite straight forward. Figure 3 depicts the conceptual workflow that

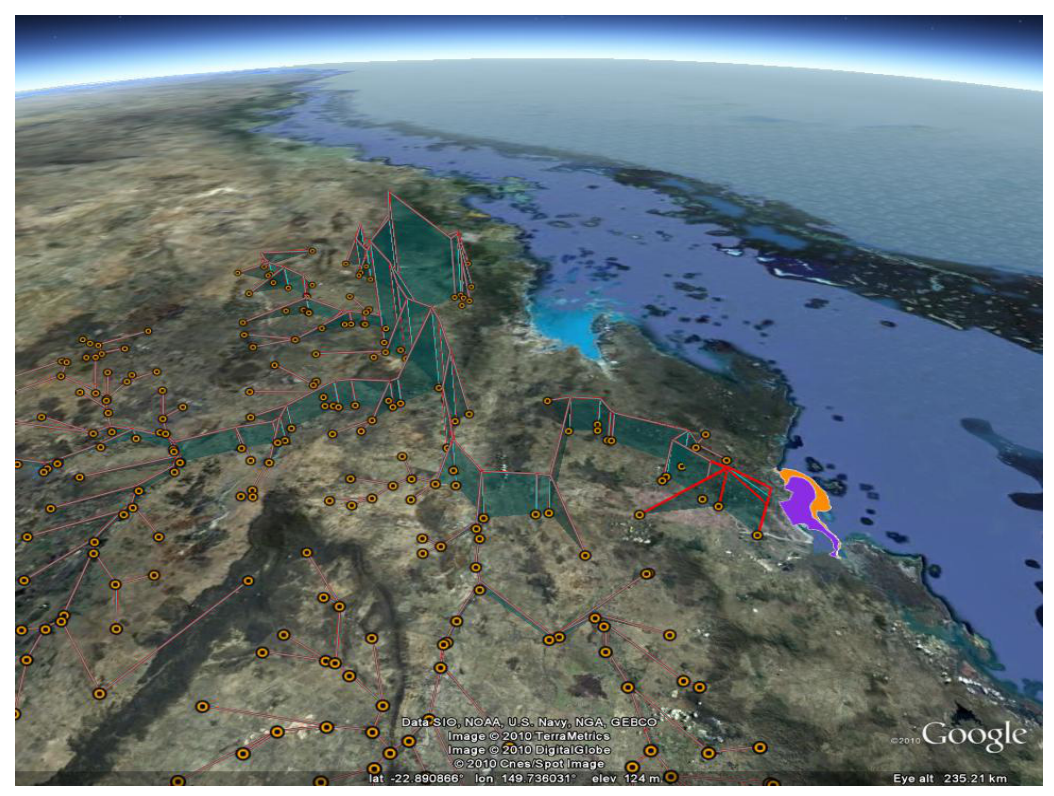

Figure 2. Example of output from the eReefs Google Earth WQVP.

would be generated in Trident. Each box in it represents a module of work, or in Trident terms, an activity. These activities, once created, were simply dragged and dropped around a canvas to create new workflows. Following steps briefly describe the process which was used to orchestrate and run the scenarios in the Pilot: (1) Open Trident and drag WaterCast Module across workflow and fill in key parameters; (2) Add Estuary Module and fill in parameters; (3) Add data format translation module to allow the communication between 
modules; (4) Add Lagoon Module to provide boundary conditions to Estuary Module; (5) Add Contours Module and specify contour intervals of interest; (6) Add KML Visualisation Tool and specify required outputs; and (7) Save and then Run the workflow. It has to be noted that prior to running the WaterCAST activity the WaterCAST software must first be manually configured (due to the way WaterCAST stores its configuration options). Also, as a Windows application Trident needs to initiate a remote session to Linux to run SHOC which runs under the Linux operating system. Details on model integration, including problems and issues associated with the workflow approach and recommendations for future development, can be found from the eReefs Pilot Report (Chen et al., 2011).

\section{SCENARIOS}

A number of scenarios were modeled as part of the eReefs Pilot to demonstrate a range of the sorts of analyses and products which would be possible with a truly integrated modelling system for the GBR catchments, in particular for Fitzroy and Burdekin (Chen et al., 2011). These are outlined in Table 1. KMLs for all scenarios were also created and visualised using the WQVP tool.

Table 1. List of modeled scenarios

\begin{tabular}{|c|c|}
\hline Catchment Scenarios & $\begin{array}{l}\text { Modifications to the base Fitzroy WaterCAST model inputs (simulation results shown as flow }\left(\mathrm{m}^{\wedge} 3 / \mathrm{s} \text { ) and/or Total }\right. \\
\text { Suspended Sediment (TSS) concentration }(\mathrm{kg} / \mathrm{s})\end{array}$ \\
\hline a. High flow scenario & $\begin{array}{l}\text { Representing extremely high flows and significantly higher TSS concentrations by simulating the catchment entirely covered } \\
\text { by urban development and with significantly large impervious surfaces. }\end{array}$ \\
\hline b. Plantation scenario & $\begin{array}{l}\text { Representing low flows and much lower sediment delivery by simulating the catchment entirely covered by forestry with } \\
\text { significantly high evapotranspiration and deep rooted woody vegetation. }\end{array}$ \\
\hline c. Grazing scenarios & 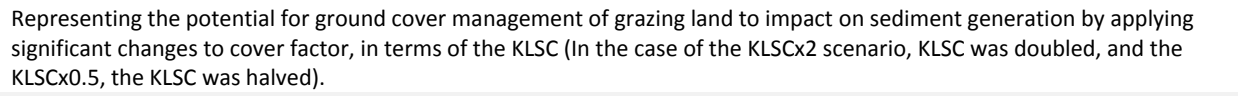 \\
\hline Estuary Scenarios & $\begin{array}{l}\text { Modifications to the base Fitzroy Estuary model, nesting within the lagoon model, using the flow outputs of Fitzroy } \\
\text { catchment scenarios as inputs (simulation results shown as salinity }(\mathrm{g} / \mathrm{kg}) \text { at the surface) }\end{array}$ \\
\hline a. High flow scenario & Representing effects of high inflows by taking the WaterCAST output for the high flow catchment scenario as input. \\
\hline b. Plantation scenario & Representing effects of low inflows by taking the WaterCAST output of the plantation catchment scenario as input. \\
\hline c. Time-shift scenario & Simulating a historical reconstruction of a low inflow conditions for the Burdekin by replacing 2008 inflows with 2005. \\
\hline d. Wind scenario & Illustrating the effect of different wind conditions on plume extent by applying low wind speed and changing wind direction. \\
\hline
\end{tabular}

\section{HISTORICAL RECONSTRUCTION}

Remote sensing reconstruction, with enhanced visibility, can help with the re-analysis of a known historical event that generated a flood plume to explore the likely extent and or ultimate fate of such a plume. Most importantly, it presents a visually appealing and intuitive way to easily share spatial information with stakeholders and the public on a common platform at any point in space and time. Reconstruction of historical events requires the combination of two approaches: firstly, reconstruction of flood plumes extents during target events using archived optical satellite remote sensing information; and secondly, reconstruction and visualisation of catchment flows using historical and modelled gauging data fed into the eReefs Google Earth Water Quality Visualisation Platform developed under the Pilot.

\subsection{Historical plume Extent Reconstruction}

This study used a GIS- and remote sensing-based methodology to map plume extents for the reappearance of historical flood events, which can help with better understanding of the exposure of GBR marine ecosystems to the terrestrial contaminants. Analysis of historical rainfall and gauge records along with a search of remote sensing archives was undertaken to allow reconstruction of several historical plume events covering the two GBR catchments, Fitzroy and Burdekin. Based on the availability of remote sensing imagery, we further analysed climate information and hydrological data to identify high flow events as our target events. Multi-spectral and multi-temporal remotely sensed imagery were digitally processed, visually interpreted and spatially delineated to map the extents of historical plumes. A two-step mapping approach was employed using available satellite data, in particular MODIS imagery. The first step is to visually interpret moderateresolution MODIS images in combination with the high-resolution Landsat ETM+ images to plot the visible boundaries of suspended sediment in plumes; and the second one is to integrate results from MODIS CDOM 
(colour dissolved organic matter) mapping (Brodie et al., 2010; Robson and Brando, 2007). The final delineation of flood-plume extents will demonstrate the ability of eReefs to communicate and visualise different water quality events over time scale. The historical reconstruction was finally displayed using the WQVP to demonstrate the potential of this methodology to communicate the past of the GBR (Figure 4).

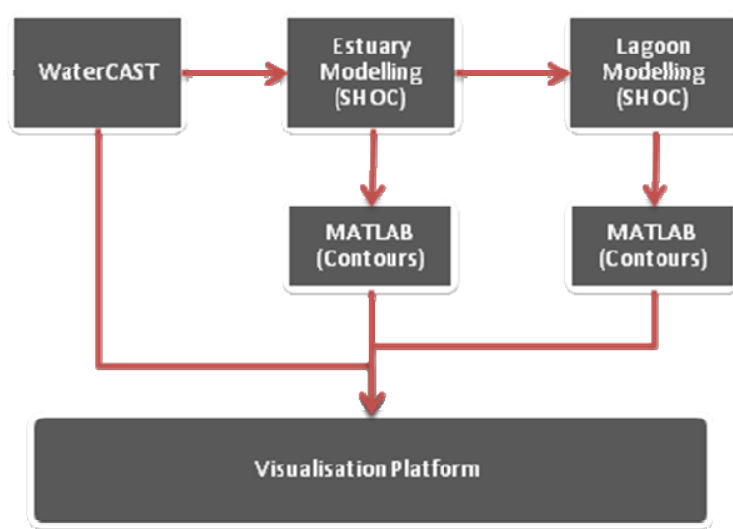

Figure 3. Conceptual workflow of orchestration.
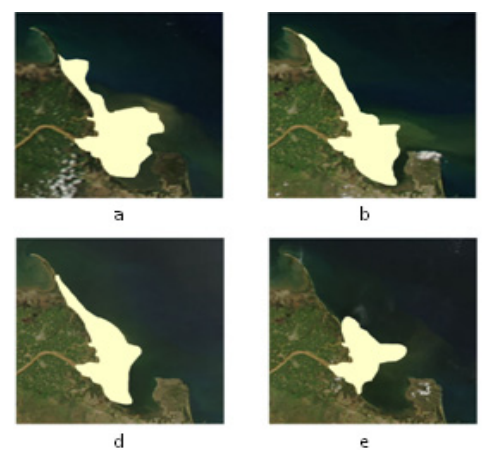
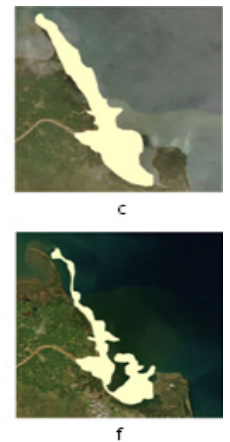

Figure 4. Snapshot frames from historical reconstruction of a flood-plume event in the Burdekin where the plume is shown changing over time (a-f: Feb 1, 2, 3, 4, 5 and 11, 2005).

\subsection{Historical Catchment Flow Reconstruction}

The reconstruction and visualisation of historical catchment flows were conducted by manually preparing data and compiling information into a XML file which transformed flow time series data from gauges into a visual time-slider representation in Google Earth. The process consisted of building a gauge node network and editing relevant data (e.g. gauge locations, flow time series, and connections between up-stream and down-stream gauges/nodes) into a XML which was then fed into the eReefs Google Earth WQVP. The node network determines the connection among the gauges. The construction of the node network was conducted by defining locations of selected gauges in ArcGIS. These nodes were then overlayed on top of the existing stream network exported from the WaterCAST modelling to decide the linkage amongst them, in terms of up-stream or down-stream connection. If the stream network is unavailable, a DEM can be used to derivethe stream network using the Hydrology Tool in ArcGIS. The reconstruction required inputs for these gauges into the XML of name, location, connection (e.g. down-stream) and corresponding flow time series. Flow time series recorded at each gauge station were collected and stored as .csv format. The compiled XML file was finally imported into the eReefs Google Earth WQVP to create a KML for the animation of historical catchment flow.

\section{REMARKS}

The eReefs pilot has demonstrated some of the possible products that would become routinely available with the development of the full eReefs vision and has immediately shown the benefits of visualising the outputs of our marine and catchment models. It has become a valuable demonstration tool for engaging investors and stakeholders in the aims of eReefs in the longer term. It has also provided an early visualisation platform for immediate rollout where the data and models exist to support the realisation of the eReefs vision. It is important to note that the eReefs Pilot project has made use of existing catchment, estuary and lagoon models which are the best currently available and reliant on having suitable data to drive them. At the moment they may not necessarily or adequately represent all of the processes, and the quality of data is extremely variable, which could result in a relatively high uncertainty in results. While the visualisation tool has been developed with the capability to display uncertainty, the assessment or estimate of uncertainty was beyond the scope of this project. The resource and time constraints on the project meant there was no opportunity for any further model validation or calibration beyond what had already been done by partners as part of prior projects. Consequently, no conclusions should be drawn from the visualisations presented by this project beyond their use for illustrating potential visualisation products that may arise from the full eReefs vision. The study has, however, paved the way for a more robust and detailed development and realisation of the fully operational eReefs in future. 


\section{ACKNOWLEDGMENTS}

The eReefs Pilot Project was funded by Telstra Corporation, the Queensland Government through the Queensland Department of Environment and Resource Management (DERM)), the Great Barrier Reef Foundation (GBRF), and CSIRO. Flow records and WaterCAST data were provided by the DERM. Products developed within the CSIRO/AIMS were used in this project: specifically, the $4 \mathrm{~km}$ grid lagoon-scale hydrodynamic model. The project also drew on previous modeling work funded by the CSIRO Water for a Healthy Country National Research Flagship: specifically, the enhanced estuary-scale hydrodynamic model grid. John Andrewartha from CSIRO Marine and Atmospheric Research (CSIRO-MAR) assisted in the development of this grid. Uwe Rosebrock (CSIRO-MAR) provided valuable technical and programming support. Marion Minjoulat-Rey from the Technical University of Ales (Ecole des Mines d'Alès) in France made a great contribution to reconstruction of historical events.

\section{REFERENCES}

Barga, R., Jackson, J., Araujo, N., Guo, D., Gautam, N., and Simmhan, Y. (2008) The Trident scientific workflow workbench," eScience '08. In: Proceedings of the IEEE Fourth International Conference. doi: 10.1109/eScience.2008.126.

Blumberg, A.F., and Herring, J. (1987) Circulation modelling using orthogonal curvilinear coordinates. In: Three-Dimensional Models of Marine and Estuarine Dynamics, Eds. J.C.J. Nihoul and B.M. Jamart, Elsevier.

Brando, V.E., Robson, B.J., Cherukuru, N.R.C., Dekker, A.G., and Webster, I.T. (2007) Towards assimilation of ocean colour satellite observation into coastal ocean biogeochemical models: the tropical Fitzroy River Estuary case study - art. no. 66850D. Assimilation of Remote Sensing and in Situ Data in Modern Numerical Weather and Environmental Prediction Models 6685.

Brodie, J., Schroeder, T., Rohde, K. Faithful, J., Masters, B., Dekker, A., and Brando, V.E. (2010) Dispersal of suspended sediments and nutrients in the Great Barrier Reef lagoon during river-discharge events: conclusions from satellite remote sensing and concurrent flood-plume sampling. Marine and Freshwater Research 61, 651-664.

Chen, Y., Seaton, S., Minchin, S., Joehnk, K. Robson, B., and Bai, Q. (2011). eReefs Pilot Report. Report to the Great Barrier Reef Foundation.

Cook, F.J., Jordan, P.W., Waters, D.K., and Rahman, J.M. (2009) WaterCAST - whole of catchment hydrology model: overview. In: Proceedings of the MODSIM 2009 International Congress on Modelling and Simulation. Modelling and Simulation Society of Australia and New Zealand.

eWater CRC, (2010). Source Catchments Scientific Reference Guide. eWater Cooperative Research Centre, Canberra.

GBR Marine Park, (2009) http://www.gbrmpa.gov.au/corp_site/about_us/great_barrier_reef_outlook_report

Herzfeld, M., Waring, J., Parslow, J., Margvelashvili, N., Sakov, P., and Andrewartha, J. (2005) SHOC: Sparse Hydrodynamic Ocean Code v1.0 Scientific Manual. CSIRO Marine and Atmospheric Research, Hobart.

Herzfeld, M.J., Andrewartha, J.R., Sakov, P., and Webster, I.T. (2006) Numerical hydrodynamic modelling of the Fitzroy Estuary. Cooperative Research Centre for the Coastal Zone, Estuary and Waterway Management, Technical Report No. 38, Australia.

Jeffrey, S.J., Carter, J.O., Moodie, K.B., and Beswick, A.R. (2001) Using spatial interpolation to construct a comprehensive archive of Australian climate data. Environmental Modelling and Software 16(4), 309-330.

Rew, R.K., Davis, G.P., Emmerson, S., and Davies H. (1997) NetCDF User's Guide for C, An Interface for Data Access, Version 3. Unidata, University Corporation for Atmospheric Research, Boulder.

Robson, B.J., and Brando, V.E. (2007) Using satellite observations to improve biogeochemical modelling of the Fitzroy River Estuary. In: Proceedings of the MODSIM 2007 International Congress on Modelling and Simulation. Modelling and Simulation Society of Australia and New Zealand.

Robson, B.J., and Brando, V.E. (2008) Simulating the response of Keppel Bay coastal waters to potential changes in sediment and nutrient loads: Final report to the Fitzroy Basin Association. Water for a Healthy Country National Research Flagship Report, CSIRO, Canberra.

Robson, B.J., Hamilton, D.P., Webster, I.T., and Chan, T. (2008) Ten steps applied to development and evaluation of process-based biogeochemical models of estuaries. Environmental Modelling and Software 23, 369-384.

Searle, R.D., and Ellis, R.J. (2009) Incorporating variable cover in erosion algorithms for grazing lands within catchment scale water quality models. In: Proceedings of the MODSIM 2009 International Congress on Modelling and Simulation. Modelling and Simulation Society of Australia and New Zealand. 